\title{
Time Delay Estimation Via Cross-Correlation in the Presence of Large Estimation Errors
}

\author{
JOHN P. IANNIELLO
}

\begin{abstract}
The estimate of the difference in time of arrival of a common random signal received at two sensors, each of which also receives uncorrelated noise, is examined for both small and large estimation errors. It is shown that as the post-integration signal-to-noise ratio decreases, the correlator exhibits a thresholding effect; that is, the probability of a large error (an anomalous estimate) increases rapidly. Approximate theoretical results for the probability of an anomaly are presented and are verified experimentally. The variance of the time delay estimate is examined for both a gated mode, in which the time delay corresponding to the correlation peak closest to the true time delay is used as the estimate of time delay, and an ungated mode, in which the time delay corresponding to the largest peak over the full range of the correlator delay times is used as the estimate. The observed variance for both modes is compared with the theoretical variance based on a small error analysis. For the gated modes, the signal-to-noise ratio below which the observed variance begins to differ significantly from the small error theory can be reliably predicted from a linearity criterion. It is shown, however, that the expected variance for the ungated mode can depart from the small error theory at a higher signal-to-noise ratio than for the gated modes; thus the variance due to anomalies can be the most important factor in determining the region of applicability of the small error analysis.
\end{abstract}

\section{INTRODUCTION}

$\mathrm{T}$ HE estimate of the difference in the time of arrival of a common random signal received at two or more sensors, each of which also receives uncorrelated noise, is a problem of considerable practical interest in underwater acoustics. One common method for estimating the time of arrival difference is to cross-correlate the two signals and to select the peak of the correlogram as the estimate of the time difference. The variance of the resulting time delay estimate has been derived by several authors [1], [2]. As discussed in [1] these results require that the estimation errors be sufficiently small for the estimate to remain within the linear region of the derivative of the signal autocorrelation function. It is also well established that the cross-correlator, with proper filtering, is the optimum time delay estimator in the sense that the variance of its estimate reaches the absolute minimum variance--the Cramer-Rao lower bound (CRLB) [1]-[3]. Again the correlator is optimum only for small estimation errors. This may be seen by noting that the cross-correlator, with proper filtering, is a maximum likelihood estimator of time delay [2]; maximum likelihood estimators, however, only reach the CRLB for nonlinear estimation problems such as the one we consider here, when estimation errors are small $[4$, p. 71$]$. Recent experi-

Manuscript received July 14, 1981; revised June 11, 1982.

The author is with the Naval Underwater Systems Center, New London, CT 06320. mental studies have verified some of these theoretical conclusions [5], [6].

Time delay estimation in the presence of large estimation errors has not been thoroughly examined. In particular it is not known under what conditions the variance of the time delay estimate begins to depart significantly from that given by the small error analysis. A rule of thumb is that when the standard deviation of the estimate is on the order of the inverse signal bandwidth, for low-pass signals, or the inverse center frequency for narrow-band signals, then typical estimates surely exceed the linear region of the derivative of the signal autocorrelation function, thus the assumption required to derive the variance expression is violated, implying that the errors will be larger than predicted. A related discussion concerning the applicability of the CRLB is given in $[4, p .70]$. This argument applies only to local errors (when estimates are near the true value), it does not address the possibility of large errors or anomalous estimates. It is appropriate to consider only local errors when a tracking gate or an a priori knowledge of the time delay dynamics can be used to limit the magnitude of the error excursion. On the other hand, when a priori information about the time delay is lacking, cross-correlators are often used ungated in the sense that any time delay in the observed range of the correlogram delay times is allowed. It is important then to understand the behavior of this ungated mode.

As we show below, a fundamentally more interesting phenomenon than a simple increase in variance occurs as the postintegration signal-to-noise ratio (SNR) is decreased; namely, below a certain post-integration SNR the probability of an anomalous estimate increases suddenly and precipitously. This is a threshold phenomenon similar to that observed in frequency modulation systems $[7$, p. 661], pulse position modulation systems (PPM) [7, p. 627], or more generally for any nonlinear estimation or communications system [4, p. 273; 7, p. 617] . As discussed by Woodward $[8$, p. 90] a threshold effect is liable to occur whenever a message of low dimensionality (in our case time delay, which is one dimensional) is "encoded" in a signal having higher dimensionality or more degrees of freedom. Thus, the threshold effect is fundamental and unavoidable.

In this paper we first present an approximate analysis for the probability of an anomalous estimate, and hence for the onset of threshold. We then describe experimental results verifying the theory, and then discuss theoretical and experimental results for the variance of the time delay estimate in both the gated and ungated modes. 


\section{Analysis for Probability of AnOMaly}

We assume that we have two sensors which receive the two random signals $x_{1}(t)$ and $x_{2}(t)$ given by

$$
\begin{aligned}
& x_{1}(t)=s(t)+n_{1}(t) \\
& x_{2}(t)=s(t-D)+n_{2}(t)
\end{aligned}
$$

where $s, n_{1}$, and $n_{2}$ are uncorrelated Gaussian random processes and $D$ is the difference in arrival time. $D$ is estimated by forming the correlator output $z(\lambda)$ via

$$
\begin{array}{rlr}
z(\lambda) & =\frac{1}{T} \int_{0}^{T} x_{1}(t) x_{2}(t+\lambda) d t, \quad 0 \leqslant \lambda<T_{0} \\
& =\frac{1}{T} \int_{0}^{T} x_{1}(t+\lambda) x_{2}(t) d t, \quad-T_{0}<\lambda<0
\end{array}
$$

and selecting the value of $\lambda$ which maximizes $z(\lambda)$ as the estimate $\hat{D}$ of $D$. We will assume that $s, n_{1}$, and $n_{2}$ have identical spectra. We also assume that a $T+T_{0}$ second record of $x_{1}(t)$ and $x_{2}(t)$ is available, although we only average for $T$ seconds. By processing in this manner we avoid end effects and can form unbiased estimates which have the same variance (for noise only inputs) at each lag value. If we processed only a $T$ second record; taper scaling $[9$, p. 182] would be required to avoid bias: Then, however, each lag estimate would have a different variance. The difference between the two methods will be small for large bandwidth time products.

To determine the probability of an anomalous estimate we follow the approximate analysis used for PPM [7, p. 627]; as for PPM the approximate analysis will be shown to agree remarkably well with experiments. We first recognize that there is a signal correlation time $T_{c}$ (to be defined exactly later) over which the signal is reasonably well correlated and beyond which the signal correlation falls to zero. Thus the observed range of lag values, $\pm T_{0}$, may be expected to contain roughly $M=2 T_{0} / T_{c}$ independent values of $z(\lambda)$, which we denote by $z_{m}$. We assume that the true delay is located at one of the time delays $\lambda_{m}$ corresponding to the $z_{m}$, say $\lambda_{0}$. We define an anomalous event as one for which the estimate of time delay is farther than $\pm T_{c} / 2$ from the true value. If we define the event $E$ as

$$
E=\left[z_{m}>z_{0} \text { for at least one } \lambda_{m}\right]
$$

then we can argue as in $[7$, p. 629] that $E$ is a reasonable approximation to what we mean by an anomaly (the validity of the approximation is to be tested by experiment). Since the $\dot{z}_{m}$ are assumed independent, the probability of the event $E$ is close to the probability of error in the communication of $M$ equally likely messages or [4, p. 262]

$$
\begin{aligned}
\left.P_{r} \text { [anomaly }\right] \simeq & P_{r}[E]=1-\int_{-\infty}^{\infty} p\left(z_{0}\right) \\
& \cdot\left[\int_{-\infty}^{z_{0}} p\left(z_{m}\right) d z_{m}\right]^{M-1} d z_{0}
\end{aligned}
$$

where $p\left(z_{0}\right)$ is the probability density function of $z_{0}$ and $p\left(z_{m}\right)$ is the probability density function for any of the $z_{m}$, all of which are assumed to have identical probability density functions.

To proceed we must find appropriate forms for $p\left(z_{0}\right)$ and $p\left(z_{m}\right)$. Since $z_{0}$ and $z_{m}$ are simply cross-correlation function estimates, their mean values and variances are known $[9, \mathrm{p}$. 183]. Thus, if the autocorrelation functions of the signal $R_{s}(\tau)$ and the noises $R_{N}(\tau)$ are given by

$$
\begin{aligned}
& R_{S}(\tau)=S \rho(\tau) \\
& R_{N}(\tau)=N \rho(\tau)
\end{aligned}
$$

where $S$ and $N$ are the signal and noise variance and $\rho(\tau)$ is the normalized correlation function of both signal and noise, and if the time bandwidth product is large, i.e., $T / T_{c} \gg 1$, then

$$
\begin{aligned}
\bar{z}_{0} & =S, \bar{z}_{m}=0 \\
\operatorname{Var}\left[z_{0}\right] & \simeq\left[(S+N)^{2}+S^{2}\right] /\left(B_{s} T\right) \\
\operatorname{Var}\left[z_{m}\right] & \simeq(S+N)^{2} /\left(B_{s} T\right) \\
B_{s} T & \equiv\left[\frac{1}{T} \int_{-\infty}^{\infty} \rho^{2}(\tau) d \tau\right]^{-1}
\end{aligned}
$$

where $B_{s}$ is the statistical bandwidth [9, p. 278].

Now assuming that $z_{0}$ and $z_{m}$ are Gaussianly distributed with means and variances given by (6), we can substitute these approximations into (4) and, after normalizing variables, we find

$$
\begin{aligned}
P_{r}[\text { anomaly }] \simeq 1 & -\int_{-\infty}^{\infty} \frac{d x}{\sqrt{2 \pi}} \exp \left[-\frac{1}{2}(x-A)^{2}\right] \\
& \cdot\left\{\int_{-\infty}^{B x} \frac{d y}{\sqrt{2 \pi}} \exp \left[-\frac{1}{2} y^{2} \mid d y\right\}^{M-1}\right.
\end{aligned}
$$

where

$$
\begin{aligned}
A & \equiv \frac{S}{\operatorname{Var}\left[z_{0}\right]^{1 / 2}}=\left(B_{S} T\right)^{1 / 2} \frac{(S / N)}{\left[(S / N)^{2}+(1+S / N)^{2}\right]^{1 / 2}} \\
B & \equiv\left[\frac{\operatorname{Var}\left[z_{0}\right]}{\operatorname{Var}\left[z_{m}\right]}\right]^{1 / 2}=\left[1+\frac{(S / N)^{2}}{(1+S / N)^{2}}\right]^{1 / 2} .
\end{aligned}
$$

Note that $A$ is the post-integration SNR and $B$ is a scaling factor which takes on values between 1 and $2^{1 / 2}$. Equation (7a) will have to be evaluated numerically.

As in the communication analogy, an approximate analytic expression establishing an upper bound on $P_{r}$ [anomaly] can be found. Thus as in $[4$, p. 264]

$$
P_{r}[\text { anomaly }] \leqslant(M-1) \int_{-\infty}^{\infty} p\left(z_{0}\right)\left[\int_{z_{0}}^{\infty} p\left(z_{m}\right) d z_{m}\right] d z_{0} .
$$

Then, again assuming that the underlying probability distributions are Gaussian with means and variances given by (6), it can be shown that

$$
P_{r}[\text { anomaly }] \leqslant(M-1) \frac{1}{\sqrt{2 \pi} C} \exp \left[-\frac{1}{2} C^{2}\right]
$$


where

$$
C \equiv\left(B_{s} T\right)^{1 / 2} \frac{S / N}{\left[(S / N)^{2}+2(1+S / N)^{2}\right]^{1 / 2}} .
$$

\section{Description of EXPERIMENT}

A computer experiment simulating the problem of interest was run by generating two unit variance, independent Gaussian random number sequences, $U_{i}$ and $V_{i}$, and combining these to generate the sequences $X_{1 i}$ and $X_{2 i}$ according to the rule [10, p. 953]

$$
\begin{aligned}
& X_{1 i}=(1+S / N)^{1 / 2} U_{i} \\
& X_{2 i}=(1+S / N)^{1 / 2}\left[r U_{i}+\left(1-r^{2}\right)^{1 / 2} V_{i}\right]
\end{aligned}
$$

where $r=(S / N) /[1+S / N]$. Thus both $X_{1}$ and $X_{2}$ have total variance $(1+S / N)$, and the correlation of the two channels, $\overline{X_{1} X_{2}}$, is $S / N$. This procedure thus simulates the problem described by (1) and (5) with $N$ in (5) set equal to unity, with $S$ in (5) set equal to $(S / N)$, and with $D=0$.

The sequences $X_{1 i}$ and $X_{2 i}$ were then low-pass filtered by a finite length Gaussian filter with sampled transfer function $h(n \Delta T)$ given by

$$
h(n \Delta T)=\left[2(\pi a)^{1 / 2}\right]^{-1} \exp \left[-(n \Delta T)^{2} /(4 a)\right], n \leqslant 16
$$

resulting in sequences with sampled autocorrelation function

$$
R(n \Delta T) \cong \frac{(1+S / N)}{2(2 \pi a)^{1 / 2}} \exp \left[-(n \Delta T)^{2} /(8 \mathrm{a})\right] .
$$

where $\Delta T$ is the time between samples ( $\Delta T$ is effectively set equal to unity below). These simulate time series with the spectral density

$$
G(2 \pi f) \cong(1+S / N) \exp \left[-2 a(2 \pi f)^{2}\right] .
$$

This form of filter was chosen since it is a low-pass filter whose autocorrelation function has no sidelobes; bandpass filters or filters with high sidelobes could introduce added complications which we do not wish to deal with now. We discuss this again briefly in the final section.

A simulated $T+T_{0}$ second record of filtered data was formed by the processing described above. With $T=L \Delta T$ and $T_{0}=P \Delta T$ this gives two, $L+P$ point records. The cross-correlation of these two sequences was estimated by dividing these $L+P$ point sequences into $(L+P) / P$ contiguous segments and proceeding via the FFT technique outlined in $[11, \mathrm{p} .560]$. This generates a correlogram estimate at lag values between $\pm P\left( \pm T_{0}\right.$ s).

To determine which events constitute an anomaly we first must decide on a reasonable definition for the correlation time $T_{C}$ of the process. We use the definition

$$
T_{C} \equiv R^{-1}(0) \int_{-\infty}^{\infty} R(\tau) d \tau
$$

Note that if the correlation function were a boxcar function with constant value in $-T_{1} / 2 \leqslant \tau \leqslant T_{1} / 2$ and zero elsewhere, then (12) would give $T_{C}=T_{1}$. If, as another example, the process had a spectrum that was uniform in $-B \leqslant f \leqslant B$ then (12) would give a value of $T_{C}=1 / 2 B$. For the process that we are considering we find, after substituting the continuous ver- sion of (11b) into (12) and evaluating the integral,

$$
T_{C}=2(2 \pi a)^{1 / 2} \text {. }
$$

Other definitions for $T_{C}$ could have been chosen. Equation (17) is reasonable and will be shown to yield satisfactory results. $M$, the number of independent values of $z(\lambda)$, is then given by

$$
M=\frac{2 T_{0}}{T_{C}}=\frac{2 P \Delta T}{2(2 \pi a)^{1 / 2}}=\frac{P}{(2 \pi)^{1 / 2} b}
$$

where

$$
a=b^{2} \Delta T^{2} .
$$

The region $R_{e}$ corresponding to a correct (nonanomalous) value of $\hat{D}$ is

$$
R_{e^{:-}} \frac{T_{C}}{2} \leqslant \hat{D} \leqslant \frac{T_{C}}{2} \text { or }-(2 \pi)^{1 / 2} b \leqslant \frac{\hat{D}}{\Delta T} \leqslant(2 \pi)^{1 / 2} b .
$$

Finally, to completely specify the problem we must choose a value for the bandwidth-time product given by (6d). For the spectrum of (11) $B_{s}=\left[2(\pi a)^{1 / 2}\right]^{-1}$ so with (14b)

$$
B_{s} T=\frac{L}{2(\pi)^{1 / 2} b} .
$$

To specify our problem we can choose $M$ and the FFT size $P$; this determines the spectrum parameter $b$ from $(14 \mathrm{~b})$. Then we must choose $L$, the total record length, to insure that $B_{s} T$ is large.

Having specified the parameters of the problem a large number (several thousand) of separate realizations of the $L+P$ point filtered sequences were generated and correlated. For each realization the lag region $\pm P\left( \pm T_{0}\right)$ was searched to find the location of the correlation peak (largest positive value). If the peak fell within $R_{e}$ then a refined estimate of the location of the peak was generated and stored using the parabolic interpolation formula

$$
\frac{\hat{D}}{\Delta T}=i_{p}+\frac{1}{2} \frac{z\left(i_{p+1}\right)-z\left(i_{p-1}\right)}{2 z\left(i_{p}\right)-z\left(i_{p+1}\right)-z\left(i_{p-1}\right)} .
$$

Here $i_{p}$ is the value of delay corresponding to the peak of the correlation function estimate and $i_{p \pm 1}$ are the adjacent delay values, separated by one sample interval $(\Delta T)$ from $i_{p}$. If $i_{p}$ did not fall within $R_{e}$ for a given realization, the estimate was counted as an anomaly. A refined estimate was still generated and stored using (17). In addition, when an anomalous event occurred, an estimate of the location of the peak value of the correlogram closest to the true value was also generated. Thus for every realization two delay estimates were generated. For nonanomalous events these estimates were identical. At the end of all the realizations the total number of anomalous events and the variances of the two delay estimates were generated. For each set of parameters $(M, b, L)$ data were generated as a function of SNR.

\section{Comparison of Theoretical and Experimental Results for Probability of ANOMAly}

A test example with parameters $M=16, b=(2 / \pi)^{1 / 2}, P=32$, $L=320$ (hence $B_{s} T=113$ ) was chosen. The theoretical results for the probability of anomaly plotted against the post-integra- 


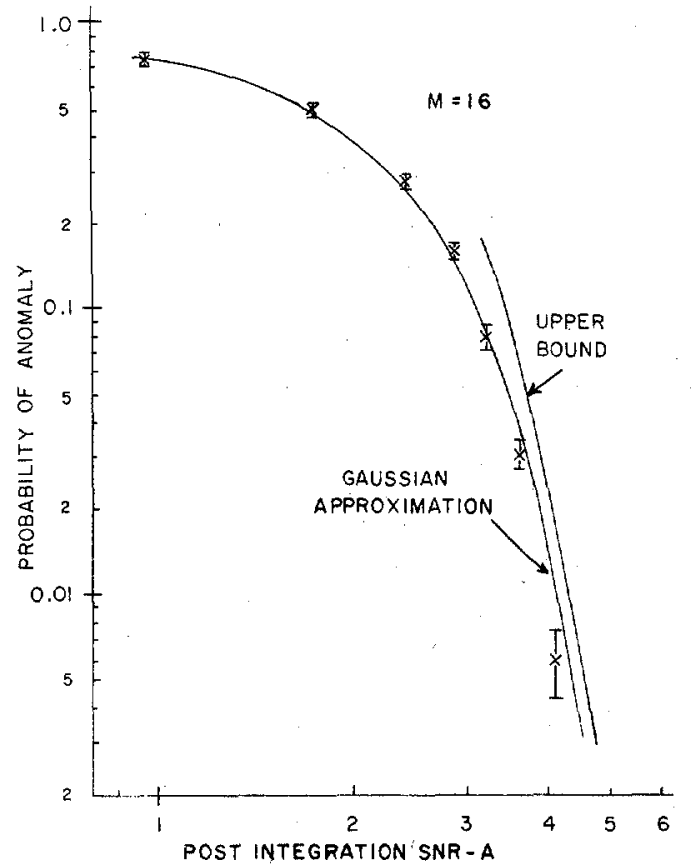

Fig. 1. Probability of anomaly versus post-integration $\mathrm{SNR}, M=16$.

tion SNR, $A$, are shown in Fig. 1. The curve labeled "Gaussian approximation" was obtained from (7). The curve labeled "Upper bound" was obtained from (9). The upper bound is close to, but does not converge to, the Gaussian approximation for the range of values shown.

The experimental results for the probability of anomaly are plotted as $x$ 's in Fig. 1. A variable number of total realizations (generally from 2000-5000) were used to obtain these data. The confidence regions indicated are 95 percent confidence intervals and are based on standard techniques for the estimation of proportions $[12$, p. 185]. The experimental results agree remarkably well with the theoretical results over the entire range of $A$ shown. The data for very small $P_{r}$ [anomaly] do appear to fall slightly below the theoretical predictions, however.

In Fig. 2 we show theoretical and experimental results for $M=4\left[\left(b=(2 / \pi)^{1 / 2}, P=8, L=320\right)\right], M=64\left[\left(b=0.4(2 / \pi)^{1 / 2}\right.\right.$, $P=128, L=128)]$ and the previous theoretical result for $M=$ 16. Both new cases have $B_{s} T=113$ as for the $M=16$ case; thus Fig. 2 reflects only the effects of varying $M$. The experimental results again confirm the applicability of the theory. From Fig. 2 we see that as $M$ is increased while holding the post-integration SNR, $A$ fixed, the probability of an anomaly increases. This is similar to the effect seen in PPM and occurs because as $M$ increases, with fixed SNR, there are simply more chances for a noise peak to exceed the peak at the true value of delay, since there are more noise bins.

$P_{r}$ [anomaly] decreases, at first, with increasing SNR. As we see from (7b), however, $A$ and hence $P_{r}$ [anomaly] eventually become independent of SNR. Thus, for $B_{s} T$ fixed, there is still a chance of making large errors even at infinite SNR. This effect occurs because the peak selecting correlator, as implemented, includes new data in the estimate of the cross-correlation at each lag value; thus it cannot be guaranteed that, even at infinite SNR, the peak value of the correlation output will

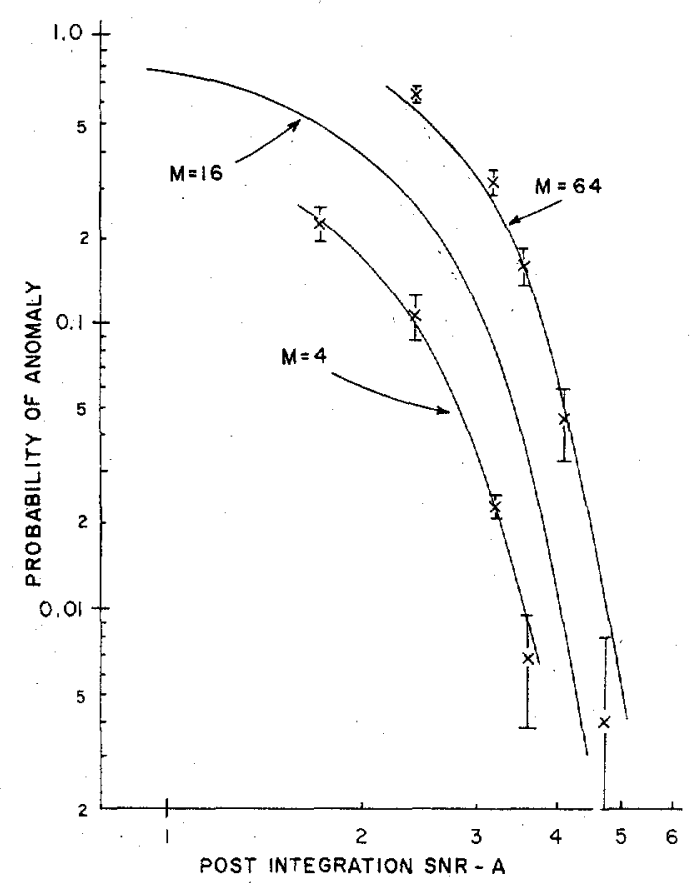

Fig. 2. Probability of anomaly versus post-integration $\mathrm{SNR}, M=4$, 16,64 .

be at the true value of time delay. It should be noted, though, that except for small $B_{s} T$, the error rate at very high $S / N$ will be quite small and hence the resulting ungated variance will be less than the variance predicted by the linear theory. This suggests that for a small $B_{s} T$ and a very large $S / N$ the peak selecting correlator may not be the best instrumentation.

\section{COMPARISON OF THEORETICAL AND EXPERIMENTAL Results For the Time Delay Estimation Variance}

Theoretical values for the variance of the time delay estimate, for small estimation errors, can be obtained from the results of [2] using the Gaussian spectral shape given by (11c). Evaluating the integrals we find

$$
\operatorname{Var}[\hat{D}]=\frac{1}{(2 \pi)\left(B_{s} T\right) B_{s}^{2}}\left[\frac{(S / N)^{2}}{1+2(S / N)}\right]^{-1} \text {. }
$$

Normalizing $\operatorname{Var}[\hat{D}]$ by $\Delta T^{2}$ and recalling the definitions $a=b^{2} \Delta T^{2}, T=L \Delta T, B_{s}^{-1}=2(\pi \mathrm{a}),(18)$ becomes

$$
\frac{\operatorname{Var}[\hat{D}]}{\Delta T^{2}}=\frac{4 \pi^{1 / 2} b^{3}}{L}\left[\frac{(S / N)^{2}}{1+2 S / N}\right]^{-1}
$$

Equation (19) is plotted, as a function of SNR, and labeled "Linear theory" in Fig. 3 using the same parameters [ $(b=$ $\left.\left.(2 / \pi)^{1 / 2}, L=320\right)\right]$ as were used for the $M=16$ example shown in Fig. 1. Experimental results for three different initial gate widths are also shown. The data labeled "Gate $\pm T_{C} / 2$ " were determined by selecting the peak value of the correlogram within the region $\pm T_{C} / 2$ (the true value is at zero delay), then interpolating using (17), and computing the variance of the resulting time delay estimates. Note that a given estimate could be outside $\pm T_{C} / 2$ after interpolation if the maximum value is not interior to $\pm T_{C} / 2$. The data labeled "Gate $\pm T_{C}$ " were computed similarly except that the initial range searched for a peak was $\pm T_{C}$. These two estimation procedures will yield identical 


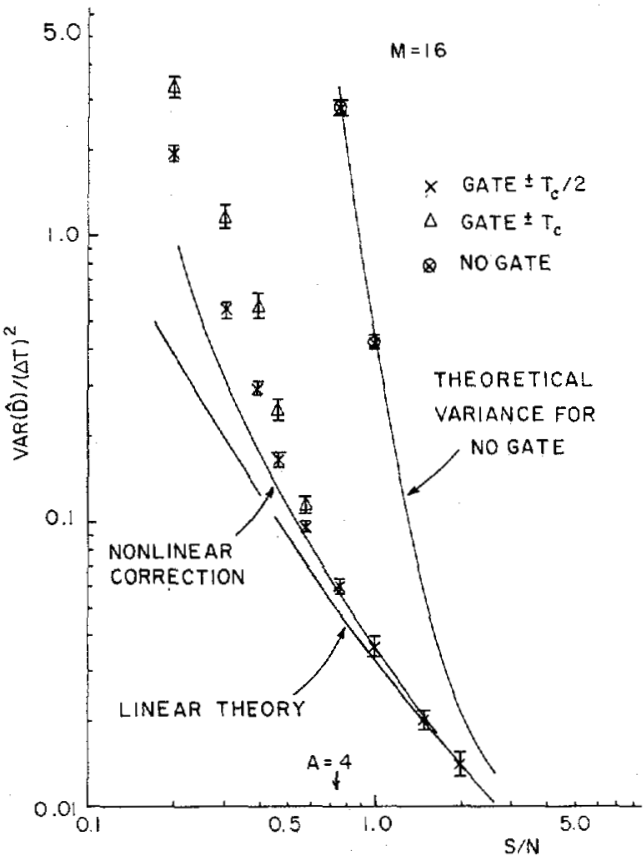

Fig. 3. Normalized variance of time delay estimate versus preintegration SNR, gated modes.

estimates except when there is a relative maximum interior to $\pm T_{C} / 2$ and a larger peak value between $T_{C} / 2$ and $T_{C}$ (or $-T_{C} / 2$ and $\left.-T_{C}\right)$. Finally, the data labeled "No gate" correspond to time delay estimates for which the entire correlogram $\left( \pm T_{0}\right)$ was searched for an initial peak.

We first discuss the results for the two gated modes. From Fig. 3 we see that for high SNR, theory and experiment agree quite well as has previously been shown [5], [6]. For $S / N=$ 0.6 or greater both gate widths gave identical results. As SNR is decreased the experimental results begin to exceed the theoretical results and at low SNR experiment and theory differ significantly. The low SNR behavior depends on the initial search gate width; as is reasonable, the larger the inital search gate the larger the variance. For SNR $=0$ the "Gate $\pm T_{C} / 2$ " data have an average normalized variance of 11.6 or a standard deviation of 0.85 times the initial gate width $\left(T_{C}=4\right)$; the "Gate $\pm T_{C}$ " data for SNR $=0$ have an average normalized variance of 14.5 or 0.48 times the initial gate width.

We now examine at what point the measured variances for the gated modes begin to depart significantly from the theoretical prediction. The error expansion used in the derivation of (18) retains only terms to the order of $d^{2} R_{s}(\tau) / d \tau^{2}[1]$. It can be shown that (18) is then valid as long as

$$
\operatorname{Var}[\hat{D}] \ll\left[\left(d^{2} R_{s} / d \tau^{2}\right) /\left(d^{4} R_{s} / d \tau^{4}\right)\right]_{\tau=0} .
$$

Equation (20) thus gives a criterion for determining when the estimates remain on the linear region of $d R_{s}(\tau) / d \tau$; For the parameters of our example the right hand side of (20) equals 0.85 ; thus we might expect (18) to be accurate as long as $\operatorname{Var}[\hat{D}] \leqslant 0.08$. From Fig. 3 we see that this is a reasonable estimate.

A simple correction to the theoretical result of (18) can be derived as follows. Equation (18) is computed from an expression of the form $[1],[4$, p. 70$]$

$$
\operatorname{Var}[\hat{D}] \equiv V_{0}^{2}=\frac{\operatorname{Var}\left(\frac{d z}{d \tau}\right)}{\left(\left.\frac{d^{2} R_{s}}{d \tau^{2}}\right|_{\tau=0}\right)^{2}} .
$$

If instead of evaluating the second derivative at $\tau=0$ we evaluate it at the value $V_{0}$ given by (21) we can find a corrected formula of the form

$$
\operatorname{Var}[D]_{C}=V_{0}^{2}\left\{\left(1-\frac{\pi}{8} V_{0}^{2}\right) \exp \left[-\pi V_{0}^{2} / 16\right]\right\}^{-2}
$$

where we have used $R(\tau)$ from $(16 \mathrm{~b}) . \operatorname{Var}[\hat{D}]_{C} / \Delta T^{2}$ is plotted in Fig. 3 and labeled "Nonlinear correction." This simple correaction is seen to work reasonably well for SNR's down to say 0.5 .

The preceeding discussion applies to the performance of the gated processing modes; to be practically usable the gated mode requires some a priori information about the true value of the time delay. If no such information is available the full correlogram delay region $\left( \pm T_{0}\right)$ must be searched. The expected variance for the ungated mode is approximately equal to the variance, given no anomaly, times the probability of no anomaly, plus the variance, given an anomaly, times the probability of an anomaly. This function is plotted and labeled "Theoretical variance for no gate" on Figs. 3 and 4. The variance, given an anomaly, is approximately $T_{0}^{2} / 3$ since this is the variance of a variable uniformly distributed in $\pm T_{0}$. The observed values are indicated by the $x$ 's in Fig. 4 , and are seen to agree quite well with the theory.

It is clear from Figs. 3 and 4 that the variance for the ungated mode departs from the linearized theory at a higher value of SNR than does the variance for the gated modes. It is of interest to have a general criterion determining which mode of processing, gated or ungated, departs from the linearized theory at the higher SNR. First, it can be shown using the criterion of (20) that, for large bandwidth time products; the gated modes begin to depart significantly from the linear theory for SNR's below the value of $(S / N)_{G} \simeq\left(30 / B_{s} T\right)^{1 / 2}$. Next we compare the value of the variance from the linear theory, evaluated at $(S / N)_{G}$, with the variance for the gated mode, also evaluated at $(S / N)_{G}$. This yields a criterion

$$
M^{3} \underset{U G}{\lessgtr} \frac{\sqrt{2}}{10 \sqrt{\pi}} C_{G} \exp \left(\frac{1}{2} C^{2}\right)
$$

where $C_{G}$ is $C$ from (9b) evaluated at $(S / N)_{G}$. Equation (23) shows that if $M^{3}$ is greater than the critical value on the right hand side then the variance for the ungated mode departs from the linear analysis at a higher SNR than does the variance for the gated mode; the opposite is true if $M^{3}$ is less than the right hand side. Hence, as stated earlier the larger the value of $M$ the more likely is anomalous behavior to be important. If $B_{s} T$ is very large then $C_{G} \simeq(15)^{1 / 2}$, independent of SNR, and the criterion becomes simply

$$
\stackrel{G}{\stackrel{U}{S}} 8 .
$$




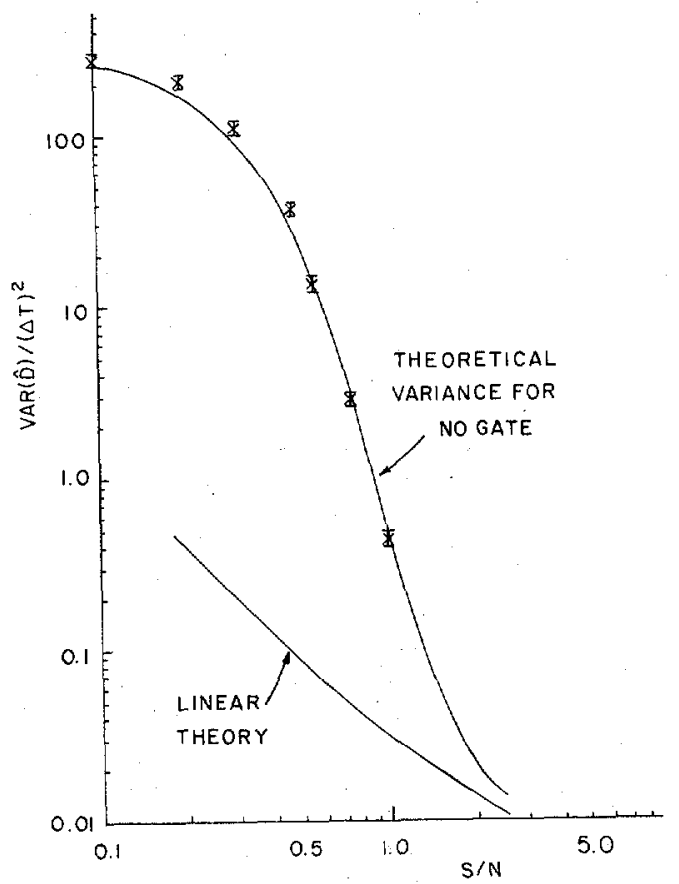

Fig. 4. Normalized variance of time delay estimate versus preintegration SNR, ungated mode.

\section{Final Comments}

The experimental results shown here were obtained using a low-pass spectrum shose autocorrelation function has no sidelobes. Signals with narrow-band spectra or with large sidelobes will have a greater probability of an anomalous estimate due to the relatively large values of the autocorrelation function at time delays removed from zero. Such spectra will have to be considered individually. One would expect the mean square error for the ungated mode to be greater than that shown here at the higher SNR's due to the effect of the sidelobes or narrowband peaks (assuming the same bandwidth). At the lower SNR's these effects will probably not be so important and hence the results will be similar to those given here.

For small estimation errors, the standard of comparison for a given instrumentation is the CRLB. Further, it is known that cross-correlators with appropriate filters achieve the CRLB. [For the Gaussian signal and noise spectra used here, however, the CRLB, found after inserting the optimum filters, is zero, since an integral over all frequency is specified. This, of course, is physically unrealistic since some other noise process will eventually become important and limit performance.] For large estimation errors other bounds such as the Barankin bound [13] or the Ziv-Zakai bound [14] are more appropriate. These bounds must be evaluated and compared with the ungated mean-square estimation error as in Fig. 4 , to establish how nearly optimum the cross-correlator is in the presence of large estimation errors.

\section{ACKNOWLEDGMENT}

I wish to thank P. Abraham, P. Cable, C. Carter, A. Nuttall, J. Pearson, and E. Weinstein for their comments and assistance at various stages of this work, and A. J. Meloney and A. J. Brault for typing the manuscript.

\section{REFERENCES}

[1] V. H. MacDonald and P. M. Schultheiss, "Optimum passive bearing estimation in a spatially incoherent noise environment," $J A S A$, vol. 46, pp. 37-43, July 1969.

[2] W. R. Hahn, "Optimum signal processing for passive sonar range and bearing estimation," JASA, vol. 58, pp. 201-207, July 1975.

[3] C. H. Knapp and G. C. Carter, "The generalized correlation method for estimation of time delay," IEEE Trans. Acoust., Speech, Signal Processing, vol. ASSP-24, pp. 320-327, Aug. 1976.

[4] H. L. Van Trees, Detection, Estimation and Modulation Theory. New York: Wiley, 1978.

[5] J. C. Hassab and R. E. Boucher, "A quantitative study of optimum and sub-optimum filters in the generalized correlator," in Proc. 1979 IEEE Int. Conf. A coust., Speech, Signal Processing, 1979, pp. 124-127.

[6] K. Scarbrough, N. Ahmed, and G. C. Carter, "On the simulation of a class of time-delay estimation algorithms," IEEE Trans. Acoust., Speech, Signal Processing, vol. ASSP-29, pp. 534-539, June 1981.

[7] J. M. Wozencraft and I. M. Jacobs, Principles of Communication Engineering. New York: Wiley, 1965.

[8] P. M. Woodward, Probability and Information Theory, with Applications to Radar. Oxford: Pergamon, 1953.

[9] J. S. Bendat and A. G. Piersol, Random Data: Analysis and Measurement Procedures. New York: Wiley-Interscience, 1971.

[10] M. Abramowitz and I. A. Stegun, Handbook of Mathematical Functions. New York: Dover, 1965.

[11] A. V. Oppenheim and R. W. Schafer, Digital Signal Processing. Englewood Cliffs, NJ: Prentice-Hall, 1975.

[12] 1. Miller and J. E. Freund, Probability and Statistics for Engineers. Englewood Cliffs, NJ: Prentice-Hall, 1965.

[13] S. K. Chow and P. M. Schultheiss, "Delay estimation using narrowband processes," IEEE Trans. Acoust., Speech, Signal Processing, vol. ASSP-29, pp. 478-484, June 1981.

[14] A. Weiss and E. Weinstein, "Passive time delay estimation from ambiguity prone signals-Part 1 ," submitted to IEEE Trans. Acoust., Speech, Signal Processing, 1982.

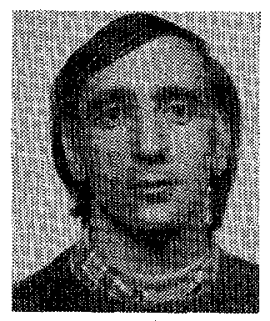

John P. Ianniello received the B.Eng. degree in electricial engineering from Yale University, New Haven, CT, in 1965, the M.S. degree in electrical engineering from the University of Connecticut in 1968 , and the Ph.D. in physical oceanography from the University of Connecticut, Storrs, in 1977. He joined the staff of NUSC, New London, CT, in 1965. Since that time he has conducted analytic and experimental investigations of passive sonar system performance, analytical studies of estuarine circulation, and experimental studies of near bottom estuarine turbulence. His recent research has centered on the large error performance of time delay estimators for various ocean propagation modes and signal spectra. 